\title{
On the Reliability of Complex Systems with Three Dependent Components per Element
}

\author{
M. Razmkhah, Z. Saberzade \\ Department of Statistics, Ferdowsi University of Mashhad, Mashhad, Iran \\ Received: 22/02/2016, Revision received: 30/10/2016, Published online: 27/05/2017
}

\begin{abstract}
The complex system containing $n$ elements, each having three dependent components, is described. The reliability function of such systems is investigated using a trivariate binomial model. In addition, the mean residual life function of a complex system with intact components at time $t$ is derived. The results are simplified for a trivariate Farlie-Gumbel-Morgenstern family with standard exponential marginal distribution functions. The effect of various parameters on the reliability and mean residual life functions are studied via some graphical representations.
\end{abstract}

Keywords. Dependence parameter, Farlie-Gumbel-Morgenstern family, Mean residual life function, Order statistics, Trivariate binomial model.

MSC: Primary 62N05; Secondary 62G30.

\section{Introduction}

In reliability engineering, the probabilistic behavior of coherent systems consisting of $n$ components are investigated from reliability aspects. For more details about the structure of such systems, one may refer to the book by Barlow and Proschan (1975). The $k$-out-of- $n$ system is an special case of a coherent system which functions if and

Corresponding Author: Mostafa Razmkhah (razmkhah_m@um.ac.ir)

Zahra Saberzade (saberzadez@yahoo.com) 
only if at least $k$ of $n$ components function. The lifetime of this system is $X_{n-k+1: n}$, where $X_{1: n} \leq X_{2: n} \leq \cdots \leq X_{n: n}$ are the order statistics of the lifetimes of $n$ components. Obviously, the series and parallel systems are the special cases of the $k$-out-of- $n$ system, when $k=n$ and $k=1$, respectively. The expected remaining age of a system, given that it has survived until time $t$, is called the mean residual life (MRL) function which is a very important concept in reliability theory and survival analysis. Denoting the lifetime of a system by $X$, the MRL is defined as $E(X-t \mid X>t)$, which uniquely determines the distribution function of $X$. Many authors are interested in this function and its applications. Bairamov et al. (2002) studied the MRL function of the parallel system using the quantity $E\left(X_{n: n}-t \mid X_{1: n}>t\right)$, which is a different version of $E\left(X_{n: n}-t \mid X_{n: n}>t\right)$. Indeed, they considered the expected remaining life of the system under the condition that none of the components has failed at time $t$; see also, Asadi (2006) and Asadi and Bayramoglu $(2005,2006)$. Asadi and Goliforushani (2008) have extended the definition of MRL for an $(n-k+1)$-out-of- $n$ system as $E\left(X_{k: n}-t \mid X_{r: n}>t\right)$, for $1 \leq r \leq k \leq n$. Khanjari Sadegh $(2008,2011)$ studied the MRL functions of parallel and coherent systems with nonidentical components. For some recent results on this topic, one can refer to $\mathrm{Li}$ and Zhao (2006), Khaledi and Shaked (2007), Li and Zhang (2008), Li and Zhao (2008), Zhao et al. (2008), Zhao et al. (2013), Bairamov and Arnold (2008), Navarro and Hernandez (2008), Gurler and Bairamov (2009), Zhao and Balakrishnan (2009), Kochar and Xu (2010), Salehi et al. (2011), Chen and Wang (2013) and Izadkhah et al. (2014).

In all of these works, the system of interest consists of some independent components. The reliability of systems with dependent components has been recently studied by some authors. Dukhovny and Marichal (2012) investigated the reliability of systems with dependent components based on lattice polynomial description. Navarro et al. (2013) studied stochastic ordering properties for systems with dependent identically distributed components; see also, Lai and Xie (2006). However, in practical applications, one may encounter with systems consisting of independent elements, each containing two or more dependent components. In an interesting work, Bairamov (2013) studied the reliability and MRL function of complex systems with two dependent components per element. Here, we would like to extend this work to the situations in which there are three dependent components per element. Let us now extend the example presented by Bairamov (2013). Suppose that a factory unit consists of $n$ lines and the $i$ th line has three devices $A_{i}, B_{i}$ and $C_{i}(1 \leq i \leq n)$, which work in interaction between themselves. Moreover, assume that in the ith line, each device $A_{i}, B_{i}$ and $C_{i}$ produces one of three basic ingredients of the same product and to achieve the final product, it is necessary to produce certain number of the first, second and third 
ingredients. If the number of working devices $A_{1}, \ldots, A_{n}$ is less than $r_{1}$, the number of working devices $B_{1}, \ldots, B_{n}$ is less than $r_{2}$, and the number of working devices $C_{1}, \ldots, C_{n}$ is less than $r_{3}$, the system is assumed to be failed. In this paper, we study the reliability and MRL functions of such complex systems.

The rest of the paper is organized as follows. In Section 2, a system containing $n$ elements, each having three dependent components is described. To compute the reliability of such a system, a trivariate binomial model is considered in Section 3 . The reliability and the MRL of $\left(r_{1}, r_{2}, r_{3}\right)$-out-of- $n$ systems are studied in Sections 4 and 5 , respectively. Finally, some conclusions are presented in Section 6.

\section{Model Description}

Consider a system consisting of $n$ elements and assume that each element has three components $\left(A_{i}, B_{i}, C_{i}\right)$ with the corresponding lifetimes $\left(X_{i}, Y_{i}, Z_{i}\right)$, for $i=1,2, \ldots, n$. Assume that the components of the $i$ th element are dependent, i.e., $X_{i}, Y_{i}$, and $Z_{i}$ are dependent random variables with joint cumulative distribution function (cdf) $F(x, y, z)$, but the elements work independently with each other. More precisely, the random vectors $\left(X_{1}, Y_{1}, Z_{1}\right),\left(X_{2}, Y_{2}, Z_{2}\right), \ldots,\left(X_{n}, Y_{n}, X_{n}\right)$ are assumed to be independent. In such a system, we assume that the first, second and third components of each element are identically distributed with the same components of other elements. Furthermore, the first, second and third components working in the same line are connected in such a way that overload of one affects the lifetime of the others. For each component of the $i$ th $(i=1,2, \ldots, n)$ element, we introduce binary variables

$$
\begin{aligned}
& x_{i}= \begin{cases}1, & \text { if } A_{i}, \text { the first component of the } i \text { th elememt, works, } \\
0, & \text { otherwise }\end{cases} \\
& y_{i}= \begin{cases}1, & \text { if } B_{i}, \text { the second component of the } i \text { th elememt, works, } \\
0, & \text { otherwise }\end{cases} \\
& z_{i}= \begin{cases}1, & \text { if } C_{i}, \text { the third component of the } i \text { th elememt, works, } \\
0, & \text { otherwise }\end{cases}
\end{aligned}
$$

and the binary function

$$
\Phi\left(x_{1}, y_{1}, z_{1}, x_{2}, y_{2}, z_{2}, \ldots, x_{n}, y_{n}, z_{n}\right)= \begin{cases}1, & \text { if the system works } \\ 0, & \text { otherwise }\end{cases}
$$


which is called the structure function.

A system of $n$ elements each consisting of three components is called the $\left(r_{1}, r_{2}, r_{3}\right)$ out-of- $n$ system if it functions iff at least $r_{1}$ of the components $A_{1}, A_{2}, \ldots, A_{n}$ function, at least $r_{2}$ of the second components $B_{1}, B_{2}, \ldots, B_{n}$ function and at least $r_{3}$ of the third components $C_{1}, C_{2}, \ldots, C_{n}$ function. The structure function of this system is given by

$\Phi\left(x_{1}, y_{1}, z_{1}, x_{2}, y_{2}, z_{2}, \ldots, x_{n}, y_{n}, z_{n}\right)= \begin{cases}1, & \text { if } \sum_{i=1}^{n} x_{i} \geq r_{1}, \sum_{i=1}^{n} y_{i} \geq r_{2} \text { and } \sum_{i=1}^{n} z_{i} \geq r_{3}, \\ 0, & \text { otherwise. }\end{cases}$

Assume $\left(X_{r_{1}: n}, Y_{r_{2}: n}, Z_{r_{3}: n}\right)$ is the vector of multivariate $r_{1}$ th, $r_{2}$ th and $r_{3}$ th order statistics of the sample $\left(X_{1}, Y_{1}, Z_{1}\right),\left(X_{2}, Y_{2}, Z_{2}\right), \ldots,\left(X_{n}, Y_{n}, Z_{n}\right)$, i.e. $X_{1: n} \leq X_{2: n} \leq \ldots \leq$ $X_{n: n}, Y_{1: n} \leq Y_{2: n} \leq \ldots \leq Y_{n: n}$ and $Z_{1: n} \leq Z_{2: n} \leq \ldots \leq Z_{n: n}$. Then, the lifetime of the $\left(r_{1}, r_{2}, r_{3}\right)$-out-of- $n$ system is

$$
T_{r_{1}, r_{2}, r_{3}: n}=\min \left\{X_{n-r_{1}+1: n}, Y_{n-r_{2}+1: n}, Z_{n-r_{3}+1: n}\right\}
$$

with the reliability function

$$
P\left\{T_{r_{1}, r_{2}, r_{3}: n}>t\right\}=P\left\{X_{n-r_{1}+1: n}>t, Y_{n-r_{2}+1: n}>t, Z_{n-r_{3}+1: n}>t\right\} .
$$

Note 1. For more simplicity, for the special case of $r_{1}=r_{2}=r_{3}=k$, a $(k, k, k)$-out-of- $n$ system is called $k$-out-of- $n$ complex system.

\section{Reliability of $\left(r_{1}, r_{2}, r_{3}\right)$-out-of- $n$ System}

To compute the reliability function in (2.2), we first consider a trivariate binomial model. Let $E_{1}, F_{1}$ and $G_{1}$ be three dependent events with the complementary events $E_{0}=E_{1}^{c}$, $F_{0}=F_{1}^{c}$ and $G_{0}=G_{1}^{c}$, respectively. Assume that $P\left(E_{i} F_{j} G_{k}\right)=p_{i j k}(i, j, k=0,1)$, such that $\sum_{i, j, k} p_{i j k}=1$. Moreover, in a simple random sampling with replacement of size $n$, let $\xi_{1}, \xi_{2}$ and $\xi_{3}$ denote the number of occurrence of $E_{1}, F_{1}$ and $G_{1}$, respectively. Then, the joint pmf of $\left(\xi_{1}, \xi_{2}, \xi_{3}\right)$ is (see, Johnson et al. (1997), p. 141)

$$
\begin{aligned}
P\left(\xi_{1}=\right. & \left.i, \xi_{2}=j, \xi_{3}=k\right) \\
= & \sum_{a=0}^{\min \{i, j\}} \sum_{b=0}^{\min \{j, k\}} \sum_{c=0}^{\min \{i, k\}} \sum_{d=0}^{\min \{a, b, c\}} n !\{d !(a-d) !(c-d) !(i-a-c+d) !(b-d) ! \\
& \times(j-a-b+d) !(k-b-c+d) !(n-i-j-k+a+b+c-d) !\} \\
& \quad \times p_{111}^{d} p_{110}^{a-d} p_{101}^{c-d} p_{100}^{i-a-c+d} p_{011}^{b-d} p_{010}^{j-a-b+d} p_{001}^{k-b-c+d} p_{000}^{n-i-j-k+a+b+c-d} \\
= & \psi(n, i, j, k, \mathbf{p}), \text { say, }
\end{aligned}
$$


where $\mathbf{p}=\left\{p_{i j k} ; i, j, k=0,1\right\}$.

Now, let $(X, Y, Z)$ be a trivariate random variable with joint $\operatorname{cdf} F(x, y, z)$ and corresponding survival function $\bar{F}(x, y, z)=P(X>x, Y>y, Z>z)$. Furthermore, assume $\left(X_{1}, Y_{1}, Z_{1}\right),\left(X_{2}, Y_{2}, Z_{2}\right), \ldots,\left(X_{n}, Y_{n}, Z_{n}\right)$ are independent copies of $(X, Y, Z)$. The survival function of the vector of trivariate order statistics $\left(X_{r_{1}: n} Y_{r_{2}: n} Z_{r_{3}: n}\right)$ can be derived from a trivariate binomial model. Indeed, by taking $E_{1}=\left\{X_{i}>x\right\}, E_{0}=\left\{X_{i} \leq x\right\}, F_{1}=\left\{Y_{i}>\right.$ $y\}, F_{0}=\left\{Y_{i} \leq y\right\}, G_{1}=\left\{Z_{i}>z\right\}$ and $G_{0}=\left\{Z_{i} \leq z\right\}$, we get

$$
P\left\{X_{r_{1}: n}>x, Y_{r_{2}: n}>y, Z_{r_{3}: n}>z\right\}=\sum_{i=n-r_{1}+1}^{n} \sum_{j=n-r_{2}+1}^{n} \sum_{k=n-r_{3}+1}^{n} \psi(n, i, j, k, \mathbf{p}),
$$

where $\psi(n, i, j, k, \mathbf{p})$ is as defined in (3.1), for which the components of the vector $\mathbf{p}$ are as

$$
\begin{aligned}
& p_{111}=\bar{F}(x, y, z), \quad p_{110}=\bar{F}_{X, Y}(x, y)-\bar{F}(x, y, z), \\
& p_{101}=\bar{F}_{X, Z}(x, z)-\bar{F}(x, y, z), \quad p_{100}=F_{Y, Z}(y, z)-F(x, y, z), \\
& p_{011}=\bar{F}_{Y, Z}(y, z)-\bar{F}(x, y, z), \quad p_{010}=F_{X, Z}(x, z)-F(x, y, z), \\
& p_{001}=F_{X, Y}(x, y)-F(x, y, z), \quad p_{000}=F(x, y, z),
\end{aligned}
$$

where $F_{X, Y}(x, y)=P(X \leq x, Y \leq y)$ and $\bar{F}_{X, Y}(x, y)=P(X>x, Y>y)$ are the bivariate cdf and survival functions of $X$ and $Y$, respectively.

Remark 1. It is known that the marginal distribution of $\left(\xi_{1}, \xi_{2}\right)$ is a bivariate binomial distribution describing a fourfold model in which the events $E_{1}, E_{0}=E_{1}^{c}$ and at the same time $F_{1}, F_{0}=F_{1}^{c}$ occur with probabilities $P\left(E_{i} F_{j}\right)=\pi_{i j}(i, j=0,1)$, such that $\sum_{i, j} \pi_{i j}=1$. The corresponding pmf is given by (see, Johnson et al. (1997), p.125)

$$
P\left(\xi_{1}=i, \xi_{2}=j\right)=\sum_{k=\max \{0, i+j-n\}}^{\min \{i, j\}} c(n, k ; i, j) \pi_{11}^{k} \pi_{10}^{i-k} \pi_{01}^{j-k} \pi_{00}^{n-i-j+k},
$$

where

$$
c(n, k ; i, j)=\frac{n !}{k !(i-k) !(j-k) !(n-i-j+k) !} .
$$

Using (2.2) and (3.2), the reliability function of the $\left(r_{1}, r_{2}, r_{3}\right)$-out-of- $n$ system is

$$
P\left\{T_{r_{1}, r_{2}, r_{3}: n}>t\right\}=\sum_{i=r_{1}}^{n} \sum_{j=r_{2}}^{n} \sum_{k=r_{3}}^{n} \psi\left(n, i, j, k, \mathbf{p}^{*}\right),
$$


where $\mathbf{p}^{*}=\left\{p_{i j k}^{*} ; i, j, k=0,1\right\}$, such that

$$
\begin{aligned}
& p_{111}^{*}=\bar{F}(t, t, t), p_{110}^{*}=\bar{F}_{X, Y}(t, t)-\bar{F}(t, t, t), \\
& p_{101}^{*}=\bar{F}_{X, Z}(t, t)-\bar{F}(t, t, t), p_{100}^{*}=F_{Y, Z}(t, t)-F(t, t, t), \\
& p_{011}^{*}=\bar{F}_{Y, Z}(t, t)-\bar{F}(t, t, t), p_{010}^{*}=F_{X, Z}(t, t)-F(t, t, t), \\
& p_{001}^{*}=F_{X, Y}(t, t)-F(t, t, t), p_{000}^{*}=F(t, t, t) .
\end{aligned}
$$

To evaluate the probabilities $p_{i j k}^{*}(i, j, k=0,1)$, let us consider the Farlie-GumbelMorgenstern (FGM) family for the trivariate cdf of $(X, Y, Z)$. That is, by denoting the marginal cdf of $X$ by $F_{X}(\cdot)$, we assume that

$$
\begin{aligned}
F(x, y, z)= & F_{X}(x) F_{Y}(y) F_{Z}(z)\left\{1+\alpha\left(1-F_{X}(x)\right)\left(1-F_{Y}(y)\right)+\beta\left(1-F_{X}(x)\right)\left(1-F_{Z}(z)\right)\right. \\
& \left.+\gamma\left(1-F_{Y}(y)\right)\left(1-F_{Z}(z)\right)+\theta\left(1-F_{X}(x)\right)\left(1-F_{Y}(y)\right)\left(1-F_{Z}(z)\right)\right\},
\end{aligned}
$$

where $\alpha, \beta, \gamma, \theta$ are dependence parameters between $(X, Y),(X, Z),(Y, Z)$ and $(X, Y, Z)$, respectively, such that $\alpha, \beta, \gamma, \theta \in[-1,1]$. For more details, see for example, Johnson and Kotz (1975). Therefore, by determining the marginal cdfs, the reliability function of a $\left(r_{1}, r_{2}, r_{3}\right)$-out-of- $n$ system may be obtained using (3.5).

It can be shown that the reliability function of a 1-out-of- $n$ complex system under the FGM model with standard exponential marginals is

$$
\begin{aligned}
\varphi_{n}(t) & =P\left\{T_{1,1,1: n}>t\right\} \\
& =P\left\{X_{n: n}>t, Y_{n: n}>t, Z_{n: n}>t\right\} \\
& =1-\left[\left(1-e^{-t}\right)^{3}\left(1+\alpha e^{-2 t}+\beta e^{-2 t}+\gamma e^{-2 t}+\theta e^{-3 t}\right)\right]^{n}-3\left(1-e^{-t}\right)^{n} \\
& +\left(1-e^{-t}\right)^{2 n}\left(1+\alpha e^{-2 t}\right)^{n}+\left(1-e^{-t}\right)^{2 n}\left(1+\beta e^{-2 t}\right)^{n}+\left(1-e^{-t}\right)^{2 n}\left(1+\gamma e^{-2 t}\right)^{n} .
\end{aligned}
$$

Figure 1 shows the variety of the reliability function in (3.6) in terms of $t$, for $\alpha=\beta=$ $\gamma=\theta=0.5$ and $n=5,10,15$. It is seen that the reliability decreases when $t$ increases. Moreover, for fixed $t$, the reliability is increasing in terms of $n$.

To demonstrate how the dependence parameters affect the reliability of the 1-outof- $n$ system, we provide graphs of reliability function with respect to $\alpha$ (when $\beta=\gamma=$ $\theta=0.5$ ) and $\theta$ (when $\alpha=\beta=\gamma=0.5$ ), separately. The results are presented in Figure 2 , for $t=2$ and $n=10$. It is observed that the reliability of the 1-out-of- $n$ system is increasing with respect to $\alpha$, while it is decreasing on $\theta$. The behavior of the reliability function of such a system with respect to $\beta$ and $\gamma$, when other dependence parameters are fixed, is similar to that of $\alpha$, which are not presented here. 


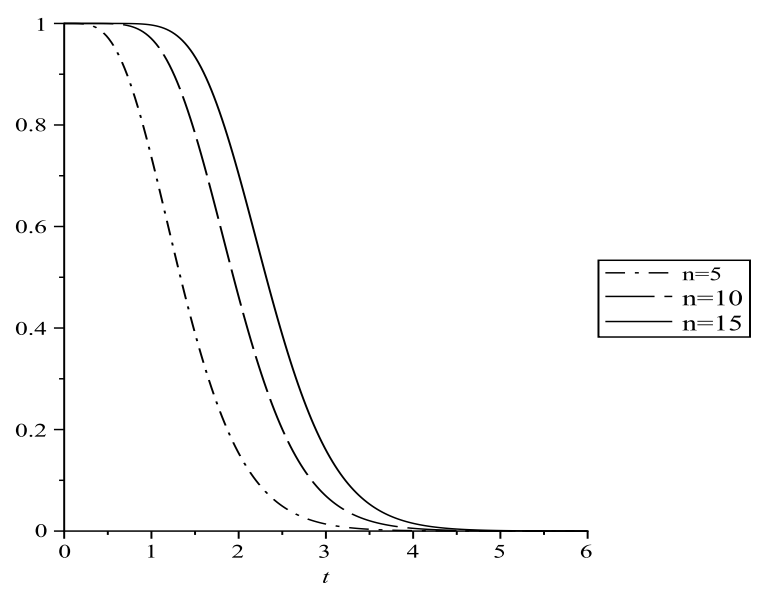

Figure 1: Graph of the reliability function $\varphi_{n}(t)$ in Equation (3.6), for $n=5,10,15$.

\section{MRL of $\left(r_{1}, r_{2}, r_{3}\right)$-out-of- $n$ System}

Here, we consider the MRL of $\left(r_{1}, r_{2}, r_{3}\right)$-out-of- $n$ system with intact components at time $t$. By conditioning on the event that none of the components have failed at time $t$, the MRL function of the $\left(r_{1}, r_{2}, r_{3}\right)$-out-of- $n$ system is defined as

$$
\begin{aligned}
\Psi_{r_{1}, r_{2}, r_{3}: n}(t) & =E\left\{T_{r_{1}, r_{2}, r_{3}: n}-t \mid X_{1: n}>t, Y_{1: n}>t, Z_{1: n}>t\right\} \\
& =\int_{0}^{\infty} S_{r_{1}, r_{2}, r_{3} ; t}(x) d x
\end{aligned}
$$

where $T_{r_{1}, r_{2}, r_{3}: n}$ is as defined in (2.1) and

$$
\begin{aligned}
& S_{r_{1}, r_{2}, r_{3} ; t}(x)=P\left(T_{r_{1}, r_{2}, r_{3}: n}-t>x \mid X_{1: n}>t, Y_{1: n}>t, Z_{1: n}>t\right) \\
& =P\left(X_{n-r_{1}+1: n}>t+x, Y_{n-r_{2}+1: n}>t+x, Z_{n-r_{3}+1: n}>t+x \mid X_{1: n}>t, Y_{1: n}>t, Z_{1: n}>t\right) \\
& =P\left(X_{n-r_{1}+1: n}>t+x, Y_{n-r_{2}+1: n}>t+x, Z_{n-r_{3}+1: n}>t+x, X_{1: n}>t, Y_{1: n}>t, Z_{1: n}>t\right) \\
& \quad \times\left\{P\left(X_{1: n}>t, Y_{1: n}>t, Z_{1: n}>t\right)\right\}^{-1} .
\end{aligned}
$$

By defining the events $M=\left\{X_{n-r_{1}+1: n} \leq t+x\right\}, N=\left\{Y_{n-r_{2}+1: n} \leq t+x\right\}, O=\left\{Z_{n-r_{3}+1: n} \leq\right.$ 

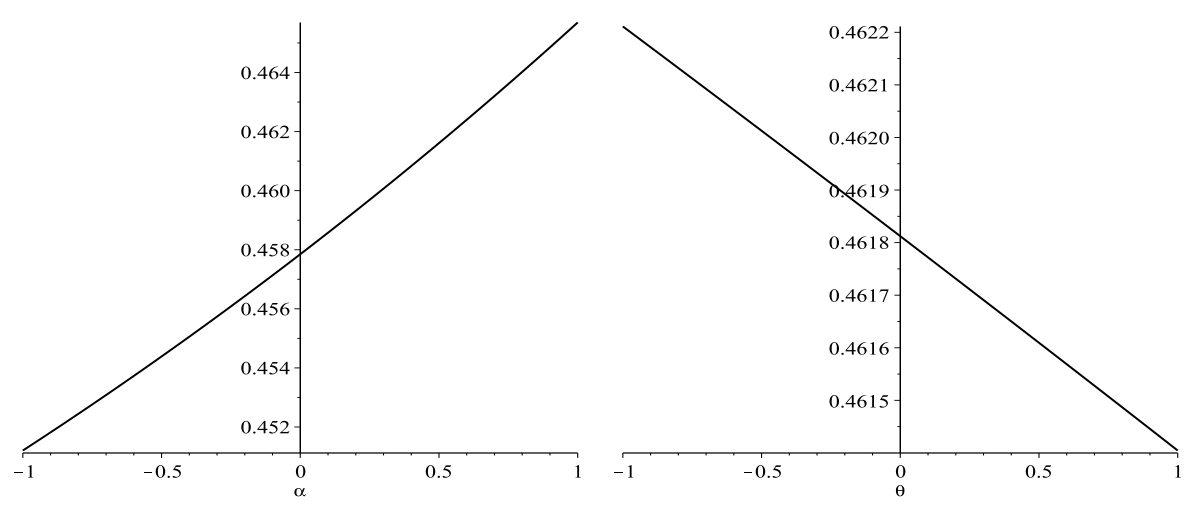

Figure 2: Graph of $\varphi_{n}(t)$ in Equation (3.6) with respect to $\alpha$ and $\theta$.

$t+x\}$ and $D=\left\{X_{1: n}>t, Y_{1: n}>t, Z_{1: n}>t\right\}$, we get

$$
\begin{aligned}
S_{r_{1}, r_{2}, r_{3} ; t}(x) & =P\left(M^{c} \cap N^{c} \cap O^{c} \cap D\right)\{P(D)\}^{-1} \\
& =\{P(D)-P(M \cap D)-P(N \cap D)-P(O \cap D)+P(M \cap N \cap D) \\
& +P(M \cap O \cap D)+P(N \cap O \cap D)-P(M \cap N \cap O \cap D)\}\{P(D)\}^{-1},
\end{aligned}
$$

where $M \cap D$ stands for intersection of the events $M$ and $D$. It is easy to see that

$$
P(D)=P\left\{X_{1: n}>t, Y_{1: n}>t, Z_{1: n}>t\right\}=[\bar{F}(t, t, t)]^{n} .
$$

For the pairwise intersection of the events $M$ and $D$, we get

$$
\begin{aligned}
& P(M \cap D)=P\left\{X_{n-r_{1}+1: n} \leq t+x, X_{1: n}>t, Y_{1: n}>t, Z_{1: n}>t\right\} \\
& =\sum_{i=n-r_{1}+1}^{n} P\left\{\text { exactly } i \text { of } X_{i} \mathrm{~s} \text { are } \leq t+x, X_{1}>t, Y_{1}>t, Z_{1}>t\right. \text {, } \\
& \left.X_{2}>t, Y_{2}>t, Z_{2}>t, \ldots, X_{n}>t, Y_{n}>t, Z_{n}>t\right\} \\
& =\sum_{i=n-r_{1}+1}^{n}\left(\begin{array}{c}
n \\
i
\end{array}\right)[P(t<X \leq x+t, Y>t, Z>t)]^{i}[P(X>x+t, Y>t, Z>t)]^{n-i} \\
& =\sum_{i=n-r_{1}+1}^{n}\left(\begin{array}{c}
n \\
i
\end{array}\right)[\bar{F}(t, t, t)-\bar{F}(t+x, t, t)]^{i}[\bar{F}(t+x, t, t)]^{n-i} \text {. }
\end{aligned}
$$


Similarly,

$$
\begin{aligned}
P(N \cap D) & =P\left\{Y_{n-r_{2}+1: n} \leq t+x, X_{1: n}>t, Y_{1: n}>t, Z_{1: n}>t\right\} \\
& =\sum_{i=n-r_{2}+1}^{n}\left(\begin{array}{l}
n \\
i
\end{array}\right)[\bar{F}(t, t, t)-\bar{F}(t, t+x, t)]^{i}[\bar{F}(t, t+x, t)]^{n-i}
\end{aligned}
$$

and

$$
\begin{aligned}
P(O \cap D) & =P\left\{Z_{n-r_{3}+1: n} \leq t+x, X_{1: n}>t, Y_{1: n}>t, Z_{1: n}>t\right\} \\
& =\sum_{i=n-r_{3}+1}^{n}\left(\begin{array}{l}
n \\
i
\end{array}\right)[\bar{F}(t, t, t)-\bar{F}(t, t, t+x)]^{i}[\bar{F}(t, t, t+x)]^{n-i} .
\end{aligned}
$$

To compute the probability of the occurrence of intersection of the events $M, N$ and $D$, we write

$$
\begin{aligned}
P(M \cap N \cap D)= & P\left\{X_{n-r_{1}+1: n} \leq t+x, Y_{n-r_{2}+1: n} \leq t+x, X_{1: n}>t, Y_{1: n}>t, Z_{1: n}>t\right\} \\
= & P\left\{X_{n-r_{1}+1: n} \leq x+t, Y_{n-r_{2}+1: n} \leq t+x, X_{1}>t, Y_{1}>t, Z_{1}>t,\right. \\
& \left.X_{2}>t, Y_{2}>t, Z_{2}>t, \ldots, X_{n}>t, Y_{n}>t, Z_{n}>t\right\} .
\end{aligned}
$$

Now, let us consider the events $E_{1}=\left\{t<X_{i} \leq t+x, Z>t\right\}, E_{0}=\left\{X_{i}>t+x, Z>t\right\}$, $F_{1}=\left\{t<Y_{i} \leq t+x, Z>t\right\}, F_{0}=\left\{Y_{i}>t+x, Z>t\right\}$ and denote by $\xi_{1}$ and $\xi_{2}$, the number of occurrence of the events $E_{1}$ and $F_{1}$ in $n$ trials with outcomes $\left(X_{1}, Y_{1}, Z_{1}\right), \ldots,\left(X_{n}, Y_{n}, Z_{n}\right)$, respectively. Then, using (3.3), we get

$$
\begin{aligned}
P(M \cap N \cap D) & =\sum_{i=n-r_{1}+1}^{n} \sum_{j=n-r_{2}+1}^{n} P\left(\xi_{1}=i, \xi_{2}=j\right) \\
& =\sum_{i=n-r_{1}+1}^{n} \sum_{j=n-r_{2}+1}^{n} \sum_{k=\max \{0, i+j-n\}}^{\min \{i, j\}} c(n, k ; i, j) \pi_{11}^{k} \pi_{10}^{i-k} \pi_{01}^{j-k} \pi_{00}^{n-i-j+k},
\end{aligned}
$$

where $c(n, k ; i, j)$ is as defined in (3.4) and

$$
\begin{aligned}
\pi_{11} & =P\{t<X \leq t+x, t<Y \leq t+x, Z>t\} \\
& =\bar{F}(t, t, t)-\bar{F}(t+x, t, t)-\bar{F}(t, t+x, t)+\bar{F}(t+x, t+x, t), \\
\pi_{10} & =P\{t<X \leq t+x, Y>t+x, Z>t\} \\
& =\bar{F}(t, t+x, t)-\bar{F}(t+x, t+x, t),
\end{aligned}
$$




$$
\begin{aligned}
\pi_{01} & =P\{X>t+x, t<Y \leq t+x, Z>t\} \\
& =\bar{F}(t+x, t, t)-\bar{F}(t+x, t+x, t), \\
\pi_{00} & =P\{X>t+x, Y>t+x, Z>t\} \\
& =\bar{F}(t+x, t+x, t) .
\end{aligned}
$$

Similarly, it can be shown that

$$
\begin{aligned}
P(M \cap O \cap D) & =\left\{X_{n-r_{1}+1: n} \leq t+x, Z_{n-r_{3}+1: n} \leq t+x, X_{1: n}>t, Y_{1: n}>t, Z_{1: n}>t\right\} \\
& =\sum_{i=n-r_{1}+1}^{n} \sum_{j=n-r_{3}+1}^{n} \sum_{k=\max \{0, i+j-n\}}^{\min \{i, j\}} c(n, k ; i, j) \rho_{11}^{k} \rho_{10}^{i-k} \rho_{01}^{j-k} \rho_{00}^{n-i-j+k},
\end{aligned}
$$

where

$$
\begin{aligned}
& \rho_{11}=\bar{F}(t, t, t)-\bar{F}(t+x, t, t)-\bar{F}(t, t, t+x)+\bar{F}(t+x, t, t+x) \\
& \rho_{10}=\bar{F}(t, t, t+x)-\bar{F}(t+x, t, t+x) \\
& \rho_{01}=\bar{F}(t+x, t, t)-\bar{F}(t+x, t, t+x) \\
& \rho_{00}=\bar{F}(t+x, t, t+x) .
\end{aligned}
$$

Furthermore,

$$
\begin{aligned}
P(N \cap O \cap D) & =\left\{Y_{n-r_{2}+1: n} \leq t+x, Z_{n-r_{3}+1: n} \leq t+x, X_{1: n}>t, Y_{1: n}>t, Z_{1: n}>t\right\} \\
& =\sum_{i=n-r_{2}+1}^{n} \sum_{j=n-r_{3}+1}^{n} \sum_{k=\max \{0, i+j-n\}}^{\min \{i, j\}} c(n, k ; i, j) \sigma_{11}^{k} \sigma_{10}^{i-k} \sigma_{01}^{j-k} \sigma_{00}^{n-i-j+k},
\end{aligned}
$$

where

$$
\begin{aligned}
& \sigma_{11}=\bar{F}(t, t, t)-\bar{F}(t, t+x, t)-\bar{F}(t, t, t+x)+\bar{F}(t, t+x, t+x), \\
& \sigma_{10}=\bar{F}(t, t, t+x)-\bar{F}(t, t+x, t+x), \\
& \sigma_{01}=\bar{F}(t, t+x, t)-\bar{F}(t, t+x, t+x), \\
& \sigma_{00}=\bar{F}(t, t+x, t+x) .
\end{aligned}
$$




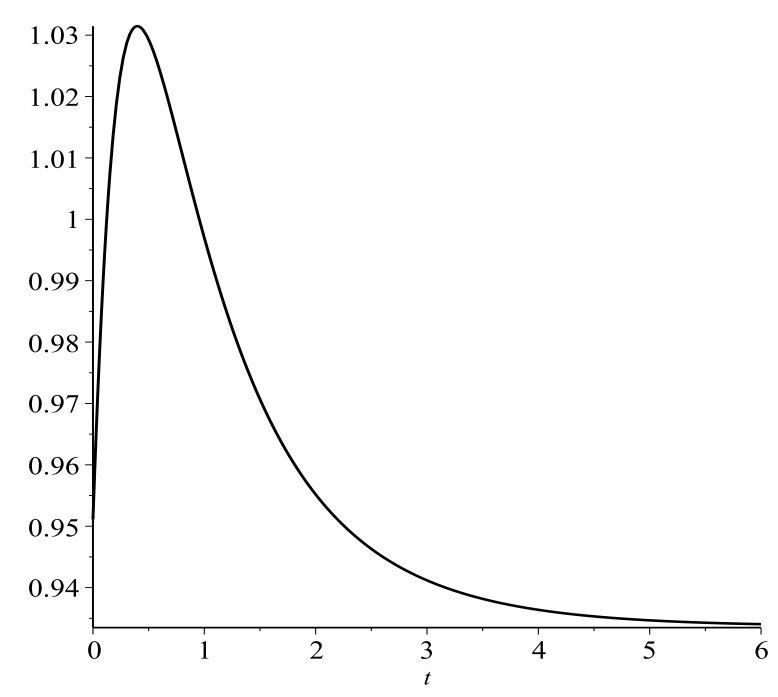

Figure 3: Graph of the MRL function $\Psi_{2,1,3: 7}(t)$, for $\alpha=\beta=\gamma=\theta=0.5$.

Finally, to compute the probability of the occurrence of event $M \cap N \cap O \cap D$, by taking $E_{1}=\left\{t<X_{i} \leq t+x\right\}, E_{0}=\left\{X_{i}>t+x\right\}, F_{1}=\left\{t<Y_{i} \leq t+x\right\}, F_{0}=\left\{Y_{i}>t+x\right\}$, $G_{1}=\left\{t<Z_{i} \leq t+x\right\}$ and $G_{0}=\left\{Z_{i}>t+x\right\}$ in a trivariate binomial model, we can write

$$
\begin{aligned}
P(M \cap N \cap O \cap D)= & P\left\{X_{n-r_{1}+1} \leq t+x, Y_{n-r_{2}+1} \leq t+x, Z_{n-r_{3}+1} \leq t+x,\right. \\
& \left.X_{1: n}>t, Y_{1: n}>t, Z_{1: n}>t\right\} \\
= & \sum_{i=n-r_{1}+1}^{n} \sum_{j=n-r_{2}+1}^{n} \sum_{k=n-r_{3}+1}^{n} \psi(n, i, j, k, v),
\end{aligned}
$$

where $\psi(n, i, j, k, v)$ is as defined in (3.1), for which the vector $v=\left\{v_{i j k} ; i, j, k=0,1\right\}$ is 
given by

$$
\begin{aligned}
v_{111} & =P\{t<X \leq t+x, t<Y \leq t+x, t<Z \leq t+x\} \\
& =\bar{F}(t, t, t)-\bar{F}(t+x, t, t)-\bar{F}(t, t+x, t)+\bar{F}(t+x, t+x, t) \\
& -\bar{F}(t, t, t+x)+\bar{F}(t+x, t, t+x)+\bar{F}(t, t+x, t+x)-\bar{F}(t+x, t+x, t+x), \\
v_{110} & =P\{t<X \leq t+x, t<Y \leq t+x, Z>t+x\} \\
& =\bar{F}(t, t, t+x)-\bar{F}(t+x, t, t+x)-\bar{F}(t, t+x, t+x)+\bar{F}(t+x, t+x, t+x), \\
v_{101} & =P\{t<X \leq t+x, Y>t+x, t<Z \leq t+x\} \\
& =\bar{F}(t, t+x, t)-\bar{F}(t+x, t+x, t)-\bar{F}(t, t+x, t+x)+\bar{F}(t+x, t+x, t+x), \\
v_{100} & =P\{t<X \leq t+x, Y>t+x, Z>t+x\} \\
& =\bar{F}(t, t+x, t+x)-\bar{F}(t+x, t+x, t+x), \\
v_{011} & =P\{X>t+x, t<Y \leq t+x, t<Z \leq t+x\} \\
& =\bar{F}(t+x, t, t)-\bar{F}(t+x, t+x, t)-\bar{F}(t+x, t, t+x)+\bar{F}(t+x, t+x, t+x), \\
v_{010} & =P\{X>t+x, t<Y \leq t+x, Z>t+x\} \\
& =\bar{F}(t+x, t, t+x)-\bar{F}(t+x, t+x, t+x), \\
v_{001} & =P\{X>t+x, Y>t+x, t<Z \leq t+x\} \\
& =\bar{F}(t+x, t+x, t)-\bar{F}(t+x, t+x, t+x), \\
v_{000} & =P\{X>t+x, Y>t+x, Z>t+x\} \\
& =\bar{F}(t+x, t+x, t+x) .
\end{aligned}
$$

After computing all of the above probabilities, the MRL of a $\left(r_{1}, r_{2}, r_{3}\right)$-out-of- $n$ system may be studied using (4.1) and (4.2). For example, by considering the FGM model for trivariate cdf of $(X, Y, Z)$ with standard exponential marginals, the MRL of a $(2,1,3)$-out-of-7 system with intact components at time $t$ is presented in Figure 3.

Also, we provide graph of the $\Psi_{2,1,3: 7}(t)$ with respect to $\alpha$ (when $\beta=\gamma=\theta=0.5$ ) and $\theta$ (when $\alpha=\beta=\gamma=0.5$ ) in Figure 4, for $t=0.3$. It is seen that by increasing the dependence parameter $\alpha$, the $(2,1,3)$-out-of-7 system becomes more reliable, i.e., the MRL is increasing in $\alpha$, but the MRL of this system decreases as $\theta$ increases. The reliability function of such a system with respect to $\beta$ and $\gamma$ behaves like $\alpha$, when other 

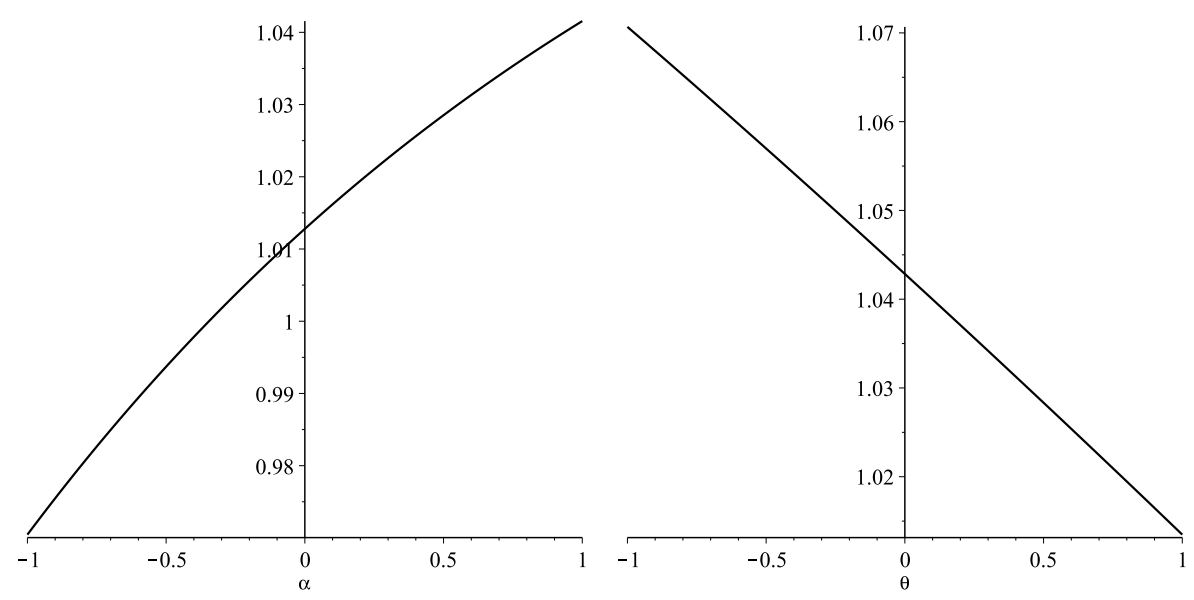

Figure 4: Graph of the MRL function $\Psi_{2,1,3: 7}(0.3)$ with respect to $\alpha$ and $\theta$.

dependence parameters are fixed, which are not presented here.

\subsection{MRL of 1-out-of- $n$ System}

Here, we study the MRL of 1-out-of- $n$ system with intact components at time $t$. Using (4.1), for the special case of $r_{1}=r_{2}=r_{3}=1$, we have

$$
\begin{aligned}
\Psi_{1,1,1: n}(t)= & \int_{0}^{\infty} S_{1,1,1 ; t}(x) d x \\
= & \int_{0}^{\infty}\{\bar{F}(t, t, t)\}^{-n}\left\{[\bar{F}(t, t, t)]^{n}-[\bar{F}(t, t, t)-\bar{F}(t+x, t, t)]^{n}\right. \\
& -[\bar{F}(t, t, t)-\bar{F}(t, t, t+x)]^{n}-[\bar{F}(t, t, t)-\bar{F}(t, t+x, t)]^{n} \\
& +[\bar{F}(t, t, t)-\bar{F}(t+x, t, t)-\bar{F}(t, t+x, t)+\bar{F}(t+x, t+x, t)]^{n} \\
& +[\bar{F}(t, t, t)-\bar{F}(t+x, t, t)-\bar{F}(t, t, t+x)+\bar{F}(t+x, t, t+x)]^{n} \\
& +[\bar{F}(t, t, t)-\bar{F}(t, t+x, t)-\bar{F}(t, t, t+x)+\bar{F}(t, t+x, t+x)]^{n} \\
& -[\bar{F}(t, t, t)-\bar{F}(t+x, t, t)-\bar{F}(t, t+x, t)+\bar{F}(t+x, t+x, t)-\bar{F}(t, t, t+x) \\
& \left.+\bar{F}(t+x, t, t+x)+\bar{F}(t, t+x, t+x)-\bar{F}(t+x, t+x, t+x)]^{n}\right\} d x .
\end{aligned}
$$

Figure 5 shows the behavior of $\Psi_{1,1,1: n}(t)$ with respect to $t$, for $\alpha=\beta=\gamma=\theta=0.5$ and $n=3,5,7$. It is seen that, for fixed $t$, the reliability function is increasing in terms of $n$. 


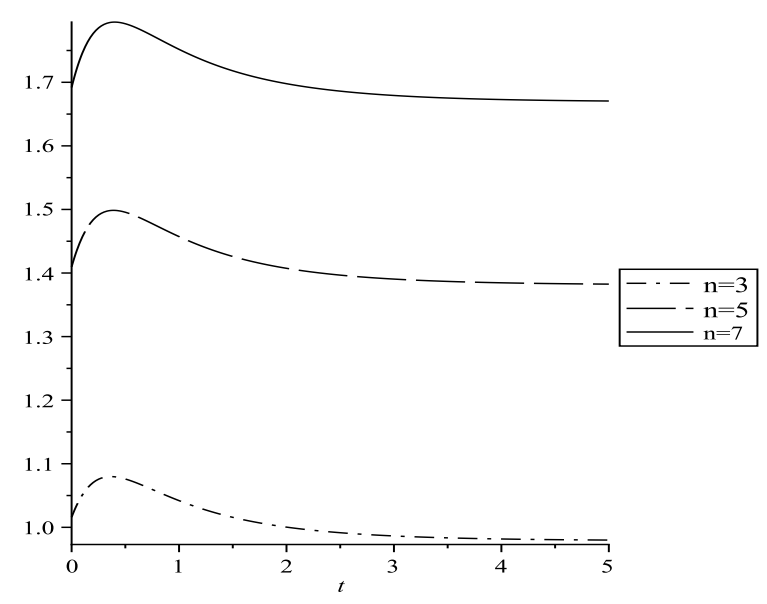

Figure 5: Graph of $\Psi_{1,1,1: n}(t)$, for $n=3,5,7$.

Also, Figure 6 demonstrates the effect of dependence parameters $\alpha$ (when $\beta=\gamma=$ $\theta=0.5$ ) and $\theta$ (when $\alpha=\beta=\gamma=0.5$ ) on the MRL of the 1-out-of- $n$ system, when $t=1.2$ and $n=7$. It is seen that the MRL function of such a system is increasing in $\alpha$, but it is decreasing in $\theta$. The behavior of the reliability function of such a system with respect to $\beta$ and $\gamma$, when other dependence parameters are fixed, is similar to that of $\alpha$, which are not presented here.

\section{Conclusion}

We considered a complex system containing $n$ elements, each having three dependent components. This paper mainly extended the paper written by Bairamov (2013) for complex systems from two dependent components per element to three components. The $\left(r_{1}, r_{2}, r_{3}\right)$-out-of- $n$ system was introduced and the reliability of such a system was studied. Also, the MRL of this complex system with intact components at time $t$ was investigated. Under an FGM family with standard exponential marginal distribution functions, some computations were performed for the special cases.

It is obvious that the results may be extended to the systems with more than three dependent components per element. In the FGM family, more other marginal distribution functions may be studied. Moreover, some other copulas, instead of FGM, may be considered as the cdf of the lifetimes of dependent components. 

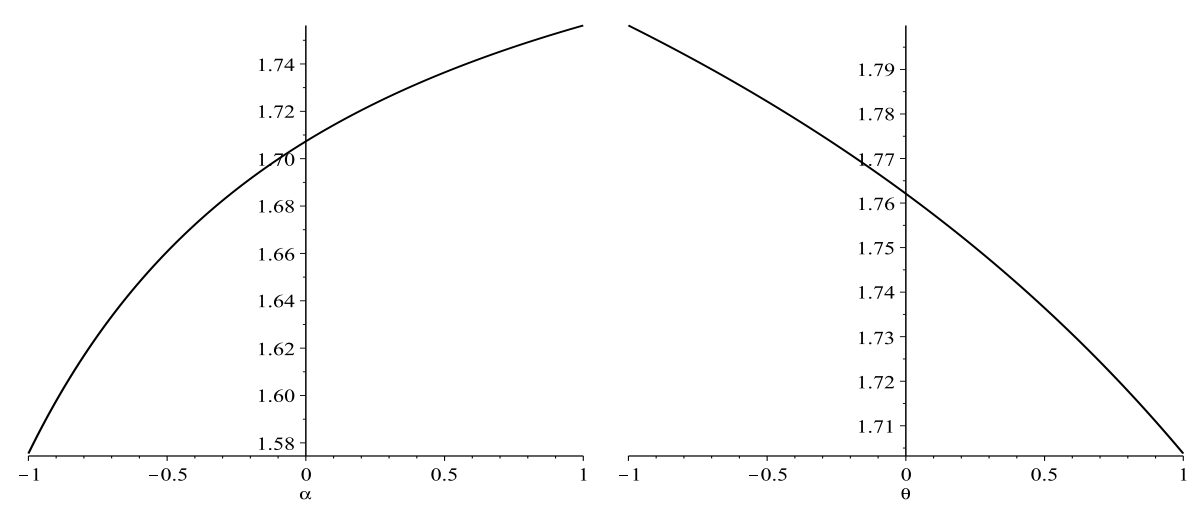

Figure 6: Graph of $\Psi_{1,1,1: 7}(1.2)$ with respect to $\alpha$ and $\theta$.

\section{References}

Asadi, M. (2006), On the mean past lifetime of the components of a parallel system. Journal of Statistical Planning and Inference, 136, 1197-1206.

Asadi, M. and Bayramoglu, I. (2005), A note on the mean residual life function of a parallel system. Communication in Statistics - Theory and Methods, 34, 475-484.

Asadi, M. and Bayramoglu, I. (2006), The mean residual life function of a k-out-of-n structure at the system level. Transactions on Reliability, 55, 314-318.

Asadi, M. and Goliforushani, S. (2008), On the mean residual life function of coherent systems. IEEE Transactions on Reliability, 57, 574-580.

Bairamov, I. (2013), Reliability and Mean Residual Life of Complex Systems With Two Dependent Components Per Element. IEEE Transactions on Reliability, 62, 276-285.

Bairamov, I., Ahsanullah, M. and Akhundov, I. (2002), A residual life function of a system having parallel or series structures. Journal of Statistical Theory and Applications, 1, 119132.

Bairamov, I. and Arnold, B. C. (2008), On the residual lifelengths of the remaining components in an $(n-k+1)$ out of $n$ system. Statistics and Probability Letters, 78, 945-952.

Barlow, R. E. and Proschan, F. (1975), Statistical Theory of Reliability and Life Testing. Holt, Rinehart and Winston, New York. 
Chen, X. and Wang, Q. (2013), Semiparametric proportional mean residual life model with covariates missing at random. Journal of Nonparametric Statistics, 25, 647-663.

Dukhoony A. and Marichal J. L. (2012), Reliability of systems with dependent Components Based on Lattice Polynomial Description. Stochastic Models, 28, 167-184.

Gurler, S. and Bairamov, I. (2009), Parallel and k-out-of-n: G systems with nonidentical components and their mean residual life functions. Applied Mathematical Modelling, 33, $1116-1125$.

Izadkhah, S., Rezaei Roknabadi, A. H. and Mohtashami Borzadaran, G. R. (2014), Aspects of the mean residual life order for weighted distributions. Statistics, 48, 851-861.

Johnson N. L. and Kotz, S. (1975), On some generalized Farlie-Gumbel-Morgenstern distributions. Communication in Statistics, 4, 415-427.

Johnson, N. L., Kotz, S. and Balakrishnan, N. (1997), Discrete Multivariate Distributions. Wiley, New York.

Khaledi, B. and Shaked, M. (2007), Ordering conditional lifetimes of coherent systems. Journal of Statistical Planning and Inference, 137, 1173-1184.

Khanjari Sadegh, M. (2008), Mean past and mean residual life functions of a parallel system with nonidentical components. Communication in Statistics - Theory and Methods, 37, 1134-1145.

Khanjari Sadegh, M. (2011), A note on the mean residual life functions of a coherent system with exchangeable or nonidentical components. Journal of Statistical Planning and Inference, 141, 3267-3275.

Kochar, S. and Xu, M. (2010), On residual lifetimes of k-out-of-n systems with nonidentical components. Probability in the Engineering and Informational Sciences, 24, 109-127.

Lai, C. D. and Xie, M. (2006). Stochastic Ageing and Dependence for Reliability. Springer, Inc.

Li, X. and Zhao, P. (2006), Some aging properties of the residual life of $k$-out-of- $n$ systems. IEEE Transactions on Reliability, 55, 535-541.

Li, X. and Zhang, Z. (2008), Some stochastic comparisons of conditional coherent systems. Applied Stochastic Models in Business and Industry, 24, 541-549. 
Li, X. and Zhao, P. (2008), Stochastic comparison on general inactivity time and general residual life of k-out-of-n systems. Communication in Statistics - Simulation and Computations, 37, 1005-1019.

Navarro, J. and Hernandez, P. J. (2008), Mean residual life functions of finite mixtures, order statistics and coherent systems. Metrika, 67, 277-298.

Navarro, J., Aguila, Y., Sordo, M. A. and Suarez-Llorens, A. (2013), Stochastic ordering properties for systems with dependent identically distributed components. Applied Stochastic Models in Business and Industry, 29, 264-278.

Salehi, E. T., Asadi, M. and Eryilmaz, S. (2011), Reliability analysis of consecutive k-outof-n systems with non-identical components lifetimes. Journal of Statistical Planning and Inference, 141, 2920-2932.

Zhao, M., Hongmei, J. and Xu, L. (2013), A note on estimation of the mean residual life function with left-truncated and right-censored data. Statistics and Probability Letters, 83, 2332-2336.

Zhao, P. and Balakrishnan, N. (2009), Stochastic comparisons and properties of conditional generalized order statistics. Journal of Statistical Planning and Inference, 139, 2920-2932.

Zhao, P., Li, X. and Balakrishnan, N. (2008), Conditional ordering of k-out-of-n systems with independent but nonidentical components. Journal of Applied Probability, 45, 1113-1125. 